\title{
BAND STRUCTURE ANALYSIS OF RECONFIGURABLE METALLIC CRYSTALS: EFFECT OF ACTIVE ELEMENTS
}

\section{A. Edalati, H. Boutayeb and T. A. Denidni}

INRS-EMT, University of Quebec

800 rue de la Gauchetiere, Montreal Quebec H5A 1K6, Canada.

\begin{abstract}
The band structure for the normal propagation of crystals formed by periodically loaded metallic wires is analyzed for different wire diameters and for various loads, which are assimilated as diodes. The diodes are simulated by using an equivalent $\mathrm{R}-\mathrm{C}$ circuit. The influences of the values of the R-C elements in On-state and Off-state are studied, and the results are compared with the band structures obtained for continuous and discontinuous-wire materials. To validate the proposed analysis, experimental results for a reconfigurable crystal are presented.
\end{abstract}

\section{INTRODUCTION}

Electromagnetic Band Gap (EBG) materials, also called Photonic Crystals, are periodic structures that offer pass-bands and stop-bands to electromagnetic waves [1-3]. They have potential applications in microwave and antenna domains, such as designing high-gain antennas [4-7], suppressing surface waves [8], increasing antenna bandwidth [9], designing Artificial Magnetic Conductors (AMC) for low-profile antennas [10], and creating controllable microwave components [11$15]$.

The objective of this paper is to describe the effect of the diodes in a reconfigurable EBG structure composed of metallic wires loaded periodically with diodes. In particular, we analyze the effect of the wire diameter and the effect of the equivalent capacitance of the diode. The band theory is an efficient method to analyze active or non-active EBG structures [1]. The dispersion characteristics of periodic structures formed by infinitely long metallic wires have been widely analyzed [1618]. By neglecting the impedance of the active elements, the band structure of the discontinuous wire medium has been studied in [19], whereas in [20,21], studies of the dispersion characteristics of crystals 
with loaded wires have been reported. However, to our knowledge, the influence of the impedance of active elements in reconfigurable EBG structures has not been studied enough. Indeed, as it is shown in this paper, the influence of the diodes is not the same for large or small wire diameter. In addition, the value of the equivalent capacitance of the diode should be taken into account in the design of a reconfigurable EBG structure, and the frequency shift due to this capacitance is evaluated in this work.

In this paper, new numerical results are presented to analyze the effect of active elements on the dispersion characteristics of reconfigurable crystals. To compute the propagation constant, a transmission line model is used, where a 2-D periodic structure (grid) of loaded wires is modeled by a T-circuit. The T-circuit parameters are expressed in terms of the S-parameters of the grid, which are computed with the Finite Difference Time domain (FDTD) method. To validate our proposed approach, experimental results are also presented and discussed.

\section{COMPUTATION OF THE PROPAGATION CONSTANT}

Figure 1 shows the 3-D periodic structure composed of periodically loaded metallic wires. The periods in $x, y$ and $z$ directions are called $P_{x}, P_{y}$ and $P_{z}$, respectively. $w$ is the width of the discontinuities and $a$ is the wire diameter. The loads are active elements, modeled by RC circuits.

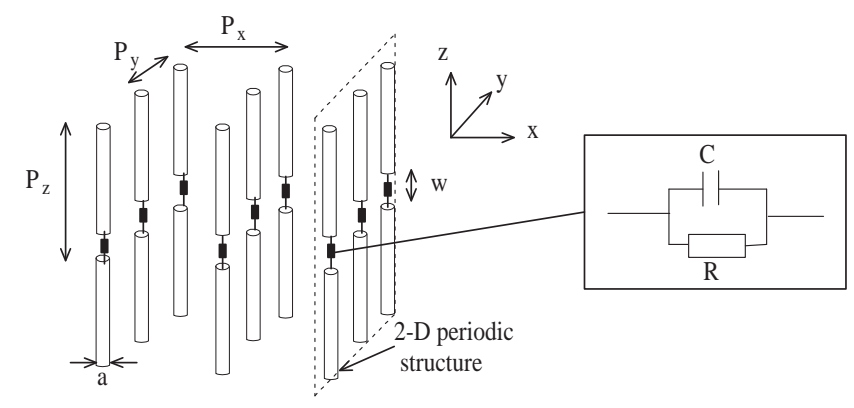

Figure 1. Infinite 3-D periodic structure of loaded metallic wires in air and equivalent RC circuits for numerical simulations.

The propagation of the transverse electric field in $\mathrm{x}$-direction is considered. To compute the propagation constant $\beta_{x}$, a transmission 
line model is used, where a 2 -D periodic structure in $y$-direction is modeled by a T-circuit [19]. $\beta_{x}$ is obtained from the following dispersion equation

$$
\cos \left(\beta_{x} P_{x}\right)=(1+Z Y) \cos \left(k P_{x}\right)+j\left(Z+\frac{Y}{2}\left(1+Z^{2}\right)\right) \sin \left(k P_{x}\right)
$$

where $k$ is the free space wave number and the coefficients $Z$ and $Y$ are derived from the reflection and transmission coefficients of the grid, $r$ and $t$ :

$$
\begin{gathered}
Y=\frac{(r-t-1)(r+t-1)}{2 t} \\
Z=\frac{-r+t-1}{r-t-1}
\end{gathered}
$$

The coefficients $r$ and $t$ were computed with a home-made Finite Difference Time Domain (FDTD) code, where image boundary conditions and a thin mesh $(\triangle=\operatorname{Period} / 80)$ were used. The scheme introduced in [22] was used to model the $R$ and $C$ elements. In the followings sections, we consider $P_{y}=P_{x}=P_{z}=P$. Because only the fundamental mode is considered, the limitation $P_{z} \leq \lambda$ is used

\section{NUMERICAL ANALYSIS}

The two first band limits for the structure with active elements in Onand Off-states are shown in Figs. 2 and 3, respectively, versus the fill factor. In On-state, we consider $R=10 \Omega$, while in Off-state we use a parallel RC circuit with $R=30 \mathrm{k} \Omega$ and different values of $C$ are tested. For comparison, the bands of structures with continuous and discontinuous wires are also presented. According to [12], for small fill factor (wire diameter), these two structures present a dual behavior in their two first bands.

From Fig. 2, it can be noted that the active element has a negligible effect in On-state when the fill factor is small. This can be explained by the fact that there is a small difference between diameters of the active element and the wire. However, for large fill factor, this difference becomes important, and the band structures of the active crystal and the continuous wire medium becomes different.

From Fig. 3, in Off-state, the active element modifies the band structure only for small wire diameter. This is due to the fact that, for large diameter, the diode capacitance is negligible compared to the capacitance of the wire. Because of the capacitance, the bands are shifted to lower frequencies by comparison with the bands obtained with the ideal discontinuous wire structure (without active elements). 


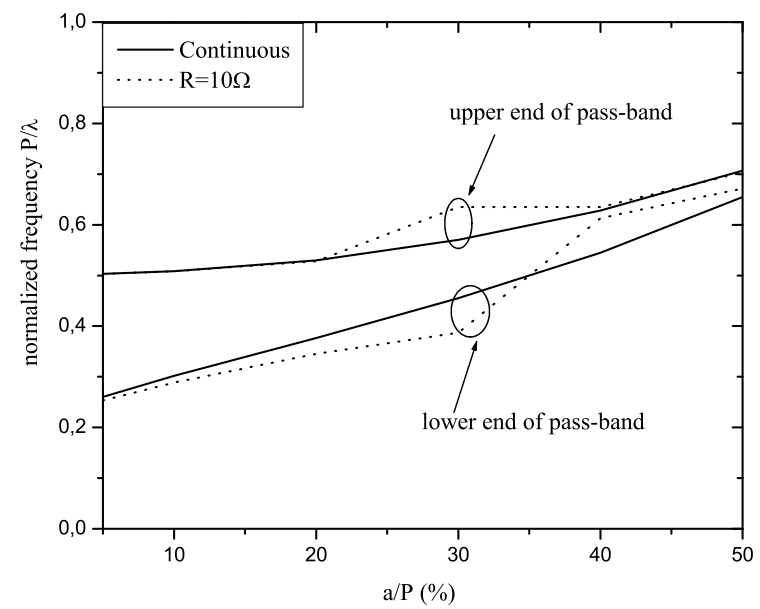

Figure 2. Two first band limits for structures with continuous wires and for wires periodically loaded with $R=10 \Omega$ versus fill factor $a / P$.

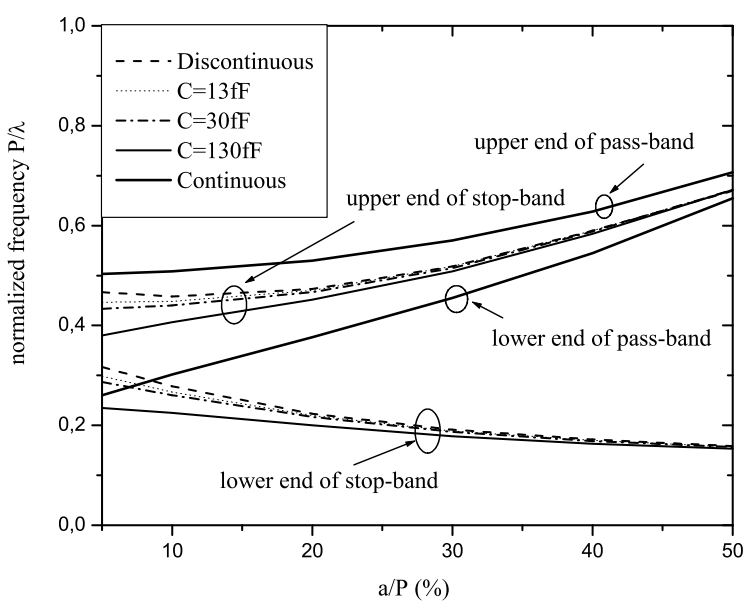

Figure 3. Two first band limits for structures with continuous, discontinuous and loaded wires with $R=30 K \Omega$, for different values of $C$, vs. fill factor $a / P$. 
This reduces the band presenting a dual behavior.

As a conclusion, for large fill factor, active elements have an effect only in On-state, whereas for small fill factor, they have an influence only in Off-state.

Now, let us consider that the fill factor is chosen small, such that only the influence of the capacitance is relevant. The shift of the lower stop-band versus the capacitance is plotted in Fig. 4. This curve is important for the design of active crystals with dual behavior of their bands, in On- or Off-states.

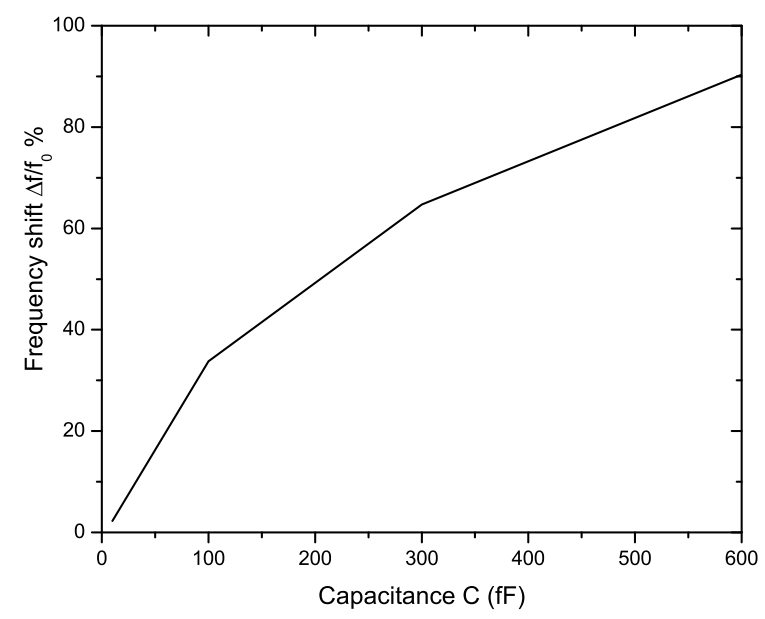

Figure 4. Frequency shift of lower stop-band versus capacitance $C$, for $a / P=10 \%$.

\section{EXPERIMENTAL RESULTS}

To validate the proposed analysis, three structures composed of five parallel sheets of metallic strips of $2 \mathrm{~mm}$ width printed on RO3003 Rogers flexible substrates with $P=20 \mathrm{~mm}$ were fabricated. The first structure is composed of continuous strips, the second one is made of discontinuous strips, and the last one contains silicon PIN diodes BAP65-03 from Philips. Strips are used instead of rods to facilitate the soldering of surface mounted diodes. The substrate is chosen flexible for future fabrication of a reconfigurable cylindrical crystal not presented here. The substrate has a permittivity of 3 , loss 
tangent of 0.0013 and a thickness of $0.254 \mathrm{~mm}$. In the discontinuous strip structure, the discontinuity width is $w=2 \mathrm{~mm}$. Figure 5 shows the photograph of the fabricated crystal. The transmission coefficients of these structures were measured in an anechoic chamber, using two horn antennas and a network analyzer. The distances between the structures and the transmitting and receiving antennas are $2 \mathrm{~m}$ and 2 $\mathrm{cm}$, respectively.

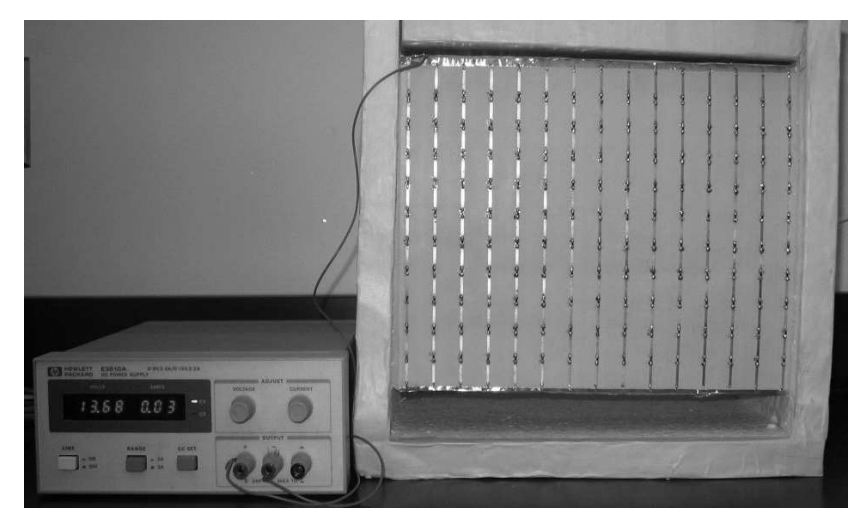

Figure 5. Photograph of the fabricated reconfigurable EBG structure.

Figures 6 (a) and (b) show the simulated and measured transmission coefficients of the structures with continuous and discontinuous strips, respectively. A good agreement is observed between theoretical and experimental data. From Fig. 6, the continuous strip structure has a stop-band from $0 \mathrm{GHz}$ to $4.5 \mathrm{GHz}$ and a pass-band from $4.5 \mathrm{GHz}$ to $7 \mathrm{GHz}$. In these two bands, the discontinuous strip structure has a dual behavior.

The structure with diodes has the same parameters than the structure with discontinuous wires expect for the added diodes. To impose the same current in each diode, resistors of $6.2 \mathrm{~K} \Omega$ are also added in both side of each strip. Note that in [11-13] inductors have been used to play this role. In On-state, a voltage supply of +13.7 Volt is used, and the current is about $0.4 \mathrm{~mA}$. For this amount of current, the diode resistance is about $1.6 \Omega$. In Off-state, the power supply is switched off, and for simulations, we considered $C=600 \mathrm{fF}$ and $R=30 K \Omega$, corresponding to the typical values given by the sheet from the manufacturer.

Figures 7 (a) and (b) show the transmission coefficient of the proposed structure, in On- and Off-states, respectively, confirming that the band structure is shifted due to the diode capacitance. The dual behavior 

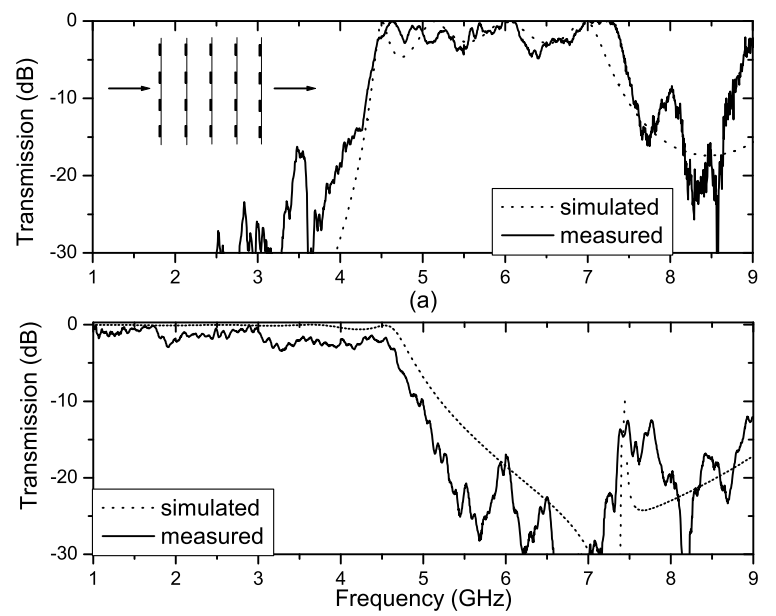

(b)

Figure 6. Transmission coefficient for a crystal with: (a) continuous strips (b) discontinuous strips.
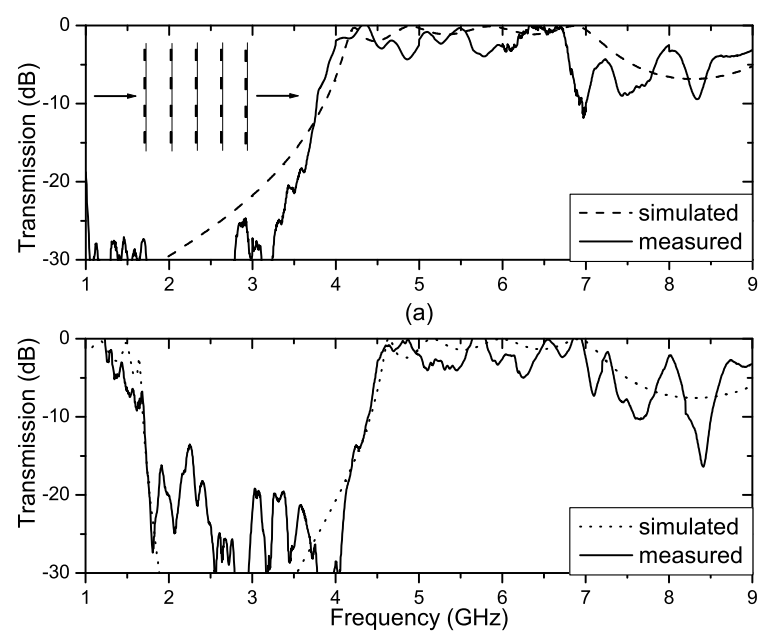

(b)

Figure 7. Transmission coefficient for an active crystal with PIN diodes: (a) On-state (b) Off-state. 
is observed only in the band $0 \mathrm{GHz}-1.8 \mathrm{GHz}$. To increase this band, one has to choose active elements that have low capacitance. According to the numerical results presented in Section 3, a value of the capacitance less than $C=100 \mathrm{fF}$ gives nearly the same dual behavior than the continuous and discontinuous strip structures.

\section{CONCLUSION}

The band structure of active metallic crystals has been studied, for different fill factors and different values of the loads. It has been shown that resistive loading has an influence only for large fill factor, and capacitive loading has an effect only for small fill factor. To validate the proposed analysis, experimental results have been presented and they show a good agreement with the theoretical ones.

\section{Acknowledgement}

This work was supported by National Science Engineering Research Council of Canada (NSERC) and Ultra-Electronics.

\section{REFERENCES}

1. Sakoda, K., Optical Properties of Photonic Crystal, ser. Opt. Sci. Berlin, Germany: Springer, 2001.

2. Boutayeb, H., K. Mahdjoubi and A.C. Tarot, "Multi-layer crystals of metallic wires: analysis of the transmission coefficient for outside and outside excitation," Progress In Electromagnetics Research, PIER 59, 299-324, 2006.

3. Dmitriev, V., "Magnetic photonic crystals with square latticegroup theoretical standpoint," Progress In Electromagnetics Research, PIER 58, 71-100, 2006.

4. Boutayeb, H., and T. A. Denidni, "Analysis and Design of a High-Gain Antenna based on Metallic Crystals," Journal of Electromagnetic Waves and Applications, Vol. 20, 599-614, 2006.

5. Pirhadi, A., and M. Hakkak, "An Analytical Investigation of the Radiation Characteristics of Infinitesimal Dipole Antenna Embedded in Partially Reflective Surfaces to Obtain High Directivity," Progress In Electromagnetics Research, PIER 65, 137-155, 2006.

6. Li, B., B. Wu, and C.-H. Liang, "Study on High Gain Circular Waveguide Array Antenna with Metamaterial Structure," Progress In Electromagnetics Research, PIER 60, 207-219, 2006. 
7. Weily, A. R., K. P. Esselle, B. C. Sanders, and T.S. Bird, "HighGain 1D EBG resonator antenna," Microw. Opt. Technol. Lett., Vol. 47, No. 2, 107-114, Oct. 2005.

8. Yang, F., and Y. Rahmat-Samii, "Microstrip Antennas Integrated With Electromagnteic Band-Gap (EBG) Structures: A Low Mutual Coupling Design for Array Applications," IEEE Trans. on Antennas and Prop., Vol. 47, No. 11, 2936-2946, Oct. 2003.

9. Pirhadi, A., M. Hakkak, and F. Keshmiri, "Using Electromagnetic Bandgap Superstrate to Enhance the Bandwidth of Probe-FED Microstrip Antenna," Progress In Electromagnetics Research, PIER 61, 215-230, 2006.

10. Sohn, J. R., K. Y. Kim, H.-S. Tae, and H. J. Lee, "Comparative Study on Various Artficial Magnetic Conductors for Low-Profile Antenna," Progress In Electromagnetics Research, PIER 61, 27$37,2006$.

11. De Lustrac, A., F. Gadot, S. Cabaret, J.M. Lourtioz, T. Brillat, A. Priou and E. Akmansoy, "Experimental demonstrattion of electrically controllable photonic crystals at centimeter wavelenghts," Applied Physics Letters, Vol. 75, No. 11, 1625-1627, Sept. 1999.

12. Lourtioz, J.M., A. De Lustrac, F. Gadot, S. Rowson, A. Chelnokov, T. Brillat, A. Ammouche, J. Danglot, O. Vanbesien, and D. Lippens, "Toward controllable photonic crystals for centimeter and millimeter wave devices," Journal of Lightwave Technology, Vol. 17, 2025-2031, Nov. 1999.

13. Djermoun, A., A. de Lustrac, J.M. Lourtioz, F. Gadot and E. Akmansoy, "Negative refraction device with electrically controllable permittivity and negative permeability," Elec. lett., Vol. 42, 223-224, Feb. 2006.

14. Boutayeb, H., A.-C. Tarot, and K. Mahdjoubi, "Focusing Characteristics of a Metallic Cylindrical Electromagnetic Band Gap Structure with Defects," Progress In Electromagnetics Research, PIER 66, 89-103, 2006.

15. Karim, M.F., A.Q. Liu, A. Alphones, A.B. Yu, "A novel reconfigurable filter using periodic structures," in Proceedings IEEE MTT-Symp., 943 - 946, Jun. 2006.

16. Simovski, C.R., M. Qiu and S. He, "The average field approach for obtaining the band structure of crystals with conducting wire inclusions," Journal of Electromagnetic Waves and Applications, Vol. 14, pp.449-468, 2000.

17. Silveirinha, M., and C. A. Fernandes, "A Hybrid method for the efficient calculation of the band structure of 3D-metallic crystal," 
IEEE Trans. Microwave Theory Tech., Vol. 52 , 889-902, March 2004.

18. Hudlicka, M., J. Machac, and I. S. Nefedov, "A Triple Wire Medium as an Isotropic Negative Permittivity Metamaterial," Progress In Electromagnetics Research, PIER 65, 233-246, 2006.

19. Boutayeb, H., T.A. Denidni, A. Sebak, and L. Talbi, "Band structure analysis of crystals with discontinuous metallic wires", IEEE Microwave Wireless Comp. Lett., Vol. 15, 484-486, July 2005.

20. Belov, P.A., C. R. Simovski, and S. A. Tretyakov, "Twodimensional electromagnetic crystals formed by reactively loaded wires," Phys. Rev. E, Vol. 66, 036610.1-036610.7, 2002.

21. Tretyakov, S. A., S. Maslovski and P.A. Belov, "An analytical model of metamaterials based on loaded wire dipole," IEEE Trans. on Antennas and Prop., Vol. 51, No. 10, 2652-2658, Oct. 2003.

22. Piket-May, M., A. Taflove and J. Baron, "FDTD modeling of digital signal propagation in 3-D circuits with passive and active loads", IEEE Trans. Microwave Theory Tech., Vol. 42, 1514 1523, Aug. 1994. 